\title{
Multiple Image Watermarking with Discrete Cosine Transform
}

\author{
Mahbuba Begum $^{1 *}$ (D), Mohammad Shorif Uddin² (i) \\ ${ }^{1}$ Department of Computer Science and Engineering, Mawlana Bhashani Science and Technology University, Tangail, Bangladesh \\ ${ }^{2}$ Department of Computer Science and Engineering, Jahangirnagar University, Dhaka, Bangladesh \\ Email: ^mahbuba327@yahoo.com,shorifuddin@gmail.com
}

How to cite this paper: Begum, M. and Uddin, M.S. (2021) Multiple Image Watermarking with Discrete Cosine Transform. Journal of Computer and Communications, 9, 88-94.

https://doi.org/10.4236/jcc.2021.93006

Received: February 24, 2021

Accepted: March 26, 2021

Published: March 29, 2021

Copyright $\odot 2021$ by author(s) and Scientific Research Publishing Inc. This work is licensed under the Creative Commons Attribution International License (CC BY 4.0).

http://creativecommons.org/licenses/by/4.0/ (c) (i) Open Access

\begin{abstract}
Multiple image watermarking is an advanced concept of singular watermarking techniques. The existing singular digital image watermarking techniques cannot obtain all the design goals, such as imperceptibility, robustness, security, and capacity simultaneously with perfection. Hence, the multiple image watermarking technique is designed that embeds several watermarks into the same host image for conveying multiple information. This paper proposed a multiple image watermarking technique with Discrete Cosine Transform (DCT) for ensuring the low computational time for embedding, encryption, decryption as well as extraction of the watermark images. Here, DCT is used to ensure the visual quality of the host image, and a random binary matrix is used to improve the security of the digital image. We have given a basic framework for multiple image watermarking. Our experimental results show satisfactory performance.
\end{abstract}

\section{Keywords}

Multiple Watermarking, Discrete Cosine Transform, Discrete Wavelet Transform, Arnold Transform

\section{Introduction}

The duplication, modification, reproduction, and distribution of digital images with a minimum cost and instant delivery are very easy without distorting the original image because of using the Internet. Besides, network technology is threatening for humans as it is developing day by day. Hence, copyright protection, image authentication, and shelter against image manipulation play an important role in facing upcoming threats for maintaining digital image information. Digital image watermarking is an alternative tool for ensuring ownership 
identification, authentication against tampering, and securing multimedia elements [1]. This technology adds the watermark image to the host data. After then, the system extracts it from the watermarked image. The invisible watermarking technology maintains the cover (or host) image beauty and quality [2].

The common singular image watermarking technology embeds single information to the host image and does not ensure high robustness and improved security. Hence, the concept of multiple watermarking has arisen. The basic multiple image watermarking technique embeds (or inserts) the multiple sets of images (either a set of different images or divides a single watermark image into multiple sets) into the host image with a watermark embedding algorithm. The watermarked image is generated as output. Then, the watermark images are extracted from the generated watermarked image, and the authenticity of the host image is verified. Here, the security key is used for encrypting the watermark images. Then, the encrypted watermark images are embedded into the host image by using the watermark embedding algorithm. The basic framework of multiple image watermark embedding is shown in Figure 1. The watermark extraction procedure is performed in a reverse way.

For ensuring an efficient system, multiple image watermarking must guarantee the basic design requirements simultaneously that are: imperceptibility, robustness, security, and capacity. However, the current research findings summarize that these basic design requirements of the system are impossible to fulfill simultaneously [3]. Hence, the proposed method is designed to increase imperceptibility and security. The multiple image watermarking technology hides and protects the data from illegal access. This technology can be used for copyright protection, integrity verification, and security of medical images.

The contributions of this paper are:

- We proposed a method that ensures the imperceptibility of the host image by using DCT.

- The method provides insignificant amount of time to embed, extract, encrypt, and decrypt the watermark images.

The paper describes the existing related literature in Section 2. The proposed methodology for multiple image watermark embedding and extraction is described

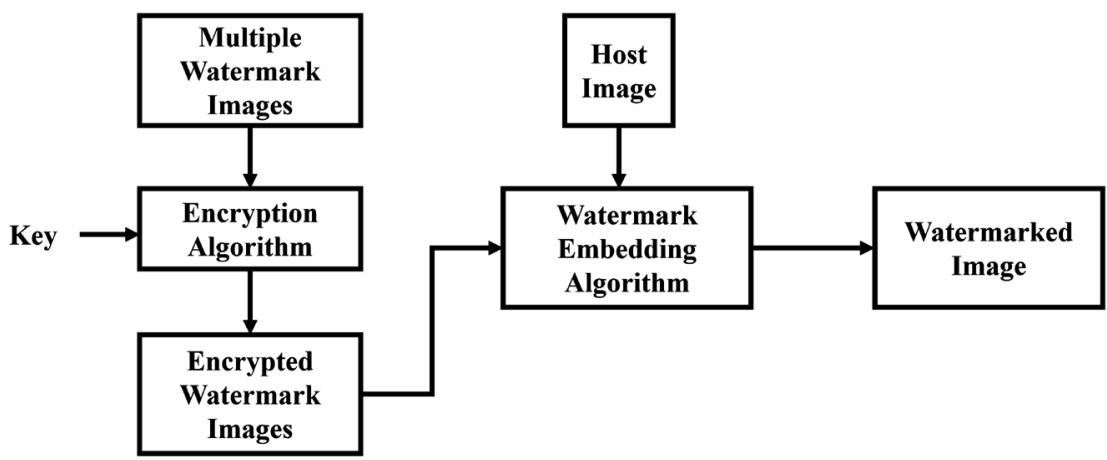

Figure 1. Basic framework for embedding of multiple watermark images. 
in Section 3. The experimental results in tabular form are described in Section 4. Finally, we conclude our study along with future work.

\section{Related Literature}

Various studies regarding multiple image watermarking concepts have already been done. Discrete Wavelet Transform (DWT) based multiple image watermarking technique is proposed for preserving the host image quality [4]. The host image is interlaced into two matrices: odd and even rows of images. For interpreting image information, the wavelet transform of the two deinterlace images is applied. Here, two watermarked images are used, and finally, these two images are extracted. This method is robust against geometric attacks and provides better quality of the watermarked image. Multiple watermarking based on hybrid DWT, SVD, and Artificial Neural Network (ANN) is proposed in scheme [5]. The optimization problem between robustness and imperceptibility is solved by applying an Artificial Bee Colony (ABC) algorithm. Another method is proposed based on Lifting Wavelet Transform (LWT), DCT, and Arnold transform [6]. Here, the watermark image is encrypted by Arnold transform. Multiple watermarks are embedded into the host (or cover) image. For embedding the watermark, the second-level (2L) LWT decomposes the original host image into sub-bands. Then, DCT is applied to LL2 and LH2, which are the low-frequency sub-bands of the host image. DCT is also applied to the two watermark images. The Arnold transformation is applied to one of the transformed watermark images. Then, the encrypted and DCT transformed watermark images are inserted into the LL2 and LH2 sub-bands, respectively. The system is robust, highly secure, and implies high data payload capacity.

Ananth et al. [7] proposed a multiple image watermarking technique based on DWT and SVD on cloud computing. Here, multiple watermarks are inserted into the host image. Their proposed method is secure and provides better imperceptibility. For selecting a suitable area for embedding the watermark, a multiple image watermarking technique is proposed based on RDWT (Redundant Discrete Wavelet Transforms), NSCT (Non-subsampled Contourlet Transform), SPIHT (Set Partitioning In Hierarchical Trees), and SVD (Singular Value Decomposition) [8]. RDWT and NSCT are appropriate for embedding the multiple watermarks. The Arnold transformation algorithm provides the enhanced security. The described system is robust and secure under a variety of attacks. In 2019 [9], another multiple image watermarking method is proposed based on Back Propagation Neural Network (BPNN) and DWT. The wavelet domain decomposes the host image, and the improved BPNN inserts the multiple watermarks into the host image. This method provides high robustness with improved visual quality. For utilizing the bandwidth and storing the medical images efficiently, a multiple image watermarking technique is proposed in the article [10]. Here, three different multiple medical watermark images are inserted into three color panes (red, green, and blue) of the host image. This embedding reduces the 
storage and transmission bandwidth. Therefore, the quality of the host image is preserved. Arnold's transformation confirms the security of the system. The proposed system is robust and secure by the experimental results.

From the above discussions, we can say that some of the existing multiple image watermarking methods ensure better imperceptibility, while some of them are robust against various attacks. But, none of the methods calculates the simulating time for embedding and extracting the watermark images. Also, they don't calculate the encryption and decryption time of the watermark images. Hence, in this paper, we have embedded multiple watermark images into the host image by using DCT. Here, DCT is used for increasing the imperceptibility and robustness of the system. Also, the system's security is ensured by using a random binary matrix. The simulation time of the system is also calculated.

\section{Proposed Methodology}

DCT is a transform domain-based watermarking scheme that ensures the imperceptibility of the watermarked image and also provides robustness under various attacks and noise addition [11]. We have added two images into a single host image by DCT fashion. Our algorithm has a total of six steps. Among these steps, four of them are key generation, preprocessing, watermark encryption, and embedding steps that are executed on the sender side. Another two steps are watermark extraction and decryption that are executed on the receiver side. In the key generation step, a random binary matrix of size [32 $\times 32]$ is generated. Preprocess means to resize the host image as [ $512 \times 512]$ and grayscale and watermark images as binary [32 $\times 32]$. For embedding the watermark images into the host image, the original host image is converted to the frequency domain by DCT. After then, the DCT bands are selected and modified. Then, the watermark embedding operation is done by the embedding algorithm, and the watermarked image is generated. The above procedure of watermark embedding is shown in Figure 2. The watermark embedding algorithm is described by Algorithm 1 . The watermark extraction and decryption steps are performed in a reverse procedure.

\section{Experimental Results}

We have used several host images (Lena, Pepper, Bandon beach, and Brandy rose) and watermark images (Panda, SEJIL, Peppers) for the experiment. The images are taken from the image database [12]. After preprocessing, the color and size of each of the host images are grayscale and $512 \times 512$, respectively. Each of the watermark images is changed to binary and $32 \times 32$ in size at the preprocessing stage. The sample host image, two watermark images, and generated watermarked image are shown in Figure 3.

The experiments are carried out with Matlab R2016a. The simulating times in seconds (s) required for key generating, preprocessing, embedding, extracting, encryption, and decryption are highlighted in Table 1. The Peak-Signal-to-Noise-Ratio 


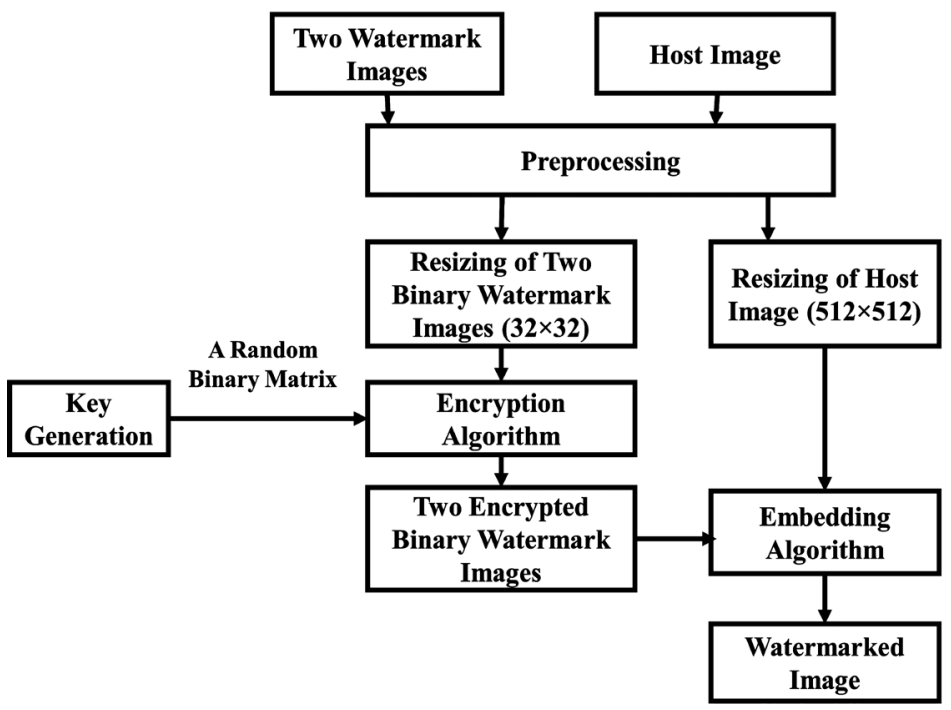

Figure 2. System flowchart of the proposed method for watermark embedding.

Algorithm 1. Watermark embedding algorithm for multiple watermark images.
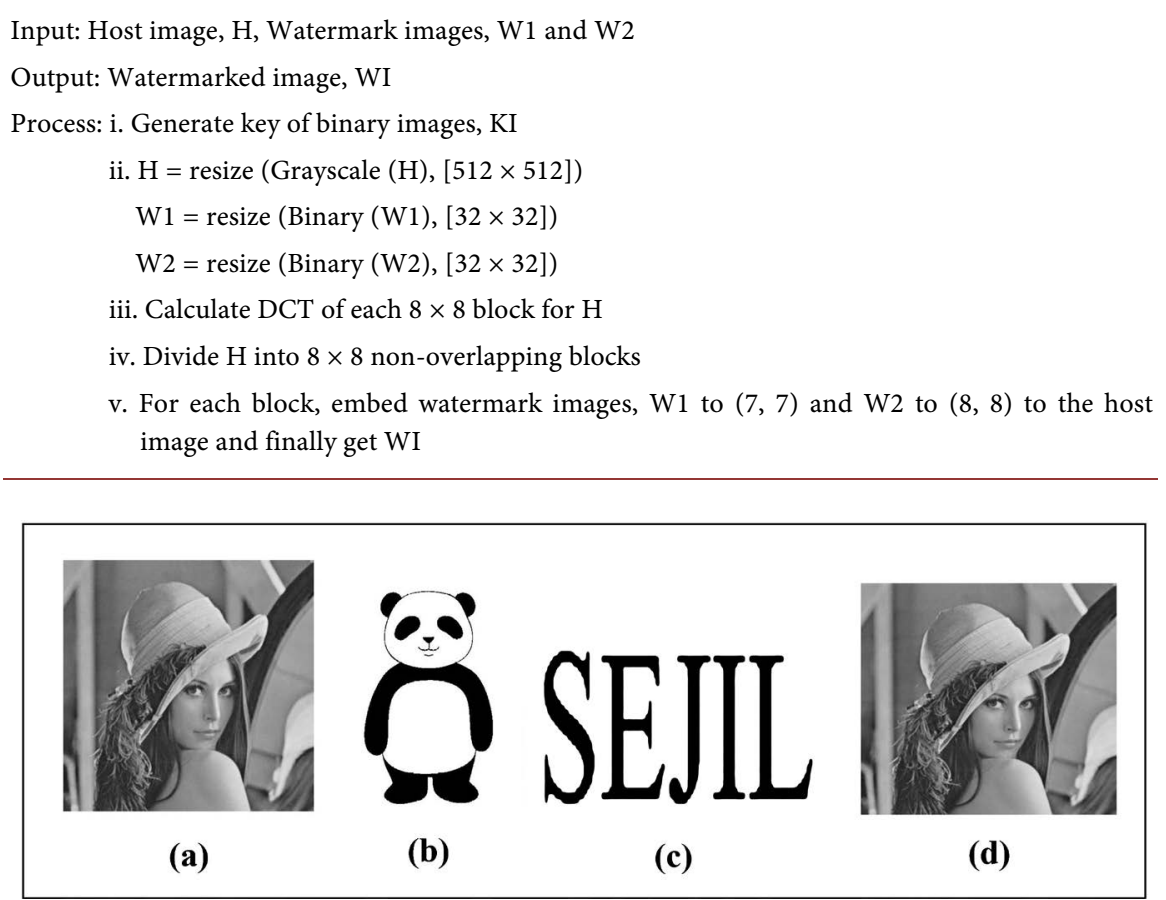

Figure 3. (a) Host image; (b) First watermark image (Panda); (c) Second watermark image; (d) Watermarked image.

Table 1. Simulating time (s) of the system and PSNR values (dB) of watermarked images.

\begin{tabular}{cccccccc}
\hline Host Images & $\begin{array}{c}\text { Key Generation } \\
\text { Time }(s)\end{array}$ & $\begin{array}{c}\text { Preprocessing } \\
\text { Time }(s)\end{array}$ & $\begin{array}{c}\text { Embedding } \\
\text { Time }(s)\end{array}$ & $\begin{array}{c}\text { Extracting } \\
\text { Time }(s)\end{array}$ & $\begin{array}{c}\text { Encryption } \\
\text { Time }(s)\end{array}$ & $\begin{array}{c}\text { Decryption } \\
\text { Time }(s)\end{array}$ & $\begin{array}{c}\text { PSNR (dB) } \\
\text { Lena }\end{array}$ \\
\hline $5.3175 \mathrm{e}^{-04}$ & 0.1339 & 0.7250 & 0.6968 & $4.3548 \mathrm{e}^{-04}$ & $4.6249 \mathrm{e}^{-04}$ & 34.6781 \\
Pepper & $2.6623 \mathrm{e}^{-04}$ & 0.2647 & 0.7000 & 0.7521 & $1.9570 \mathrm{e}^{-04}$ & $1.4909 \mathrm{e}^{-04}$ & 34.6726 \\
Bandon beach & $2.5847 \mathrm{e}^{-04}$ & 0.1591 & 0.7321 & 0.7703 & $3.9987 \mathrm{e}^{-04}$ & $3.4324 \mathrm{e}^{-04}$ & 35.2171 \\
Brandy rose & $2.6658 \mathrm{e}^{-04}$ & 0.1530 & 0.7369 & 0.6963 & $1.6432 \mathrm{e}^{-04}$ & $1.4204 \mathrm{e}^{-04}$ & 34.6835 \\
\hline
\end{tabular}


(PSNR) values in $\mathrm{dB}$ are calculated for verifying the quality of each watermarked image with the host images as references. Table 1 highlights the simulating time of the system and PSNR values of the watermarked images. It shows that the watermark encryption and decryption time are very close, and at the same time, the watermark embedding and extracting time are also close because these two processes are inverse to each other.

\section{Conclusion and Future Work}

The multiple image watermarking techniques have gained attention in today's world as they provide stronger security than singular watermarking techniques. In this paper, the multiple image watermarking system is designed with DCT to increase the imperceptibility and security for image authentication. The random binary matrix encrypts the watermark images and provides the enhanced security for the system. This multiple image watermarking technique requires insignificant amount of time for each test case like singular watermarking to simulate the system. But, the system provides a little bit of computational complexity. Here, PSNR $>30 \mathrm{~dB}$ for each test case indicates the better quality of the watermarked image. The future work can be extended by simulating the results under several single and combined attacks. Also, the performance of the proposed method will be compared with existing current state-of-the-art methods and expanded for other multimedia elements.

\section{Author Contributions}

M. B. studied, experimented, and drafted the whole paper; M. S. U. initiated the concept, supervised the study, and fine-tuned the manuscript.

\section{Funding}

No funding is received for this research.

\section{Acknowledgements}

The authors are thankful to the Information and Communication Technology Division of the Government of the People's Republic of Bangladesh for a $\mathrm{PhD}$ fellowship to Mahbuba Begum.

\section{Conflicts of Interest}

The authors declare no conflicts of interest regarding the publication of this paper.

\section{References}

[1] Begum, M. and Uddin, M.S. (2020) Digital Image Watermarking Techniques: A Review. Information, 11, 110. https://doi.org/10.3390/info11020110

[2] Begum, M. and Uddin, M.S. (2020) Analysis of Digital Image Watermarking Techniques through Hybrid Methods. Advances in Multimedia, 2020, Article ID: 7912690. 
https://doi.org/10.1155/2020/7912690

[3] Agarwal, N., Singh, A.K. and Singh, P.K. (2019) Survey of Robust and Imperceptible Watermarking. Multimedia Tools and Applications, 78, 8603-8633.

https://doi.org/10.1007/s11042-018-7128-5

[4] Sridhar, B. and Arun, C. (2012) On Secure Multiple Image Watermarking Techniques using DWT. Proceeding Third International Conference on Computing, Communication and Networking Technologies (ICCCNT'12), Coimbatore, 26-28 July 2012, 1-4. https://doi.org/10.1109/ICCCNT.2012.6395871

[5] Mohananthini, N. and Yamuna, G., (2015) Multiple Image Watermarking Technique based on Hybrid DWT, SVD and Artificial Neural Network. International Journal of Applied Engineering Research, 10, 7275-7297.

[6] Preet, C. and Aggarwal, R.K. (2017) Multiple Image Watermarking Using LWT, DCT and Arnold Transformation. International Conference on Trends in Electronics and Informatics (ICEI), Tirunelveli, 11-12 May 2017, 158-162. https://doi.org/10.1109/ICOEI.2017.8300908

[7] Ananth, C., Karthikeyan, M. and Mohananthini, N. (2018) DWT-SVD based Multiple Image Watermarking Process on Cloud Computing. International Journal of Research Granthaalayah, 6, 88-96.

https://doi.org/10.29121/granthaalayah.v6.i6.2018.1339

[8] Kumar, C., Singh, A.K., Kumar, P., Singh, R. and Singh, S. (2018) SPIHT-Based Multiple Image Watermarking in NSCT Domain. Concurrency and Computation: Practice and Experience, e4912, 1-9. https://doi.org/10.1002/cpe.4912

[9] Ananth, C., Karthikeyan, M., Mohananthini, N., Saravanan, S. and Swathisriranjani, M. (2019) Multiple Watermarking for Images using Back-Propagation Neural Network and DWT. International Journal of Engineering and Advanced Technology (IJEAT), 9, 4088-4093. https://doi.org/10.35940/ijeat.A1327.109119

[10] Anjum, R., Verma, P. and Verma, S. (2019) Multiple Image Watermarking for Efficient Storage and Transmission of Medical Images. In: Singh, M., Gupta, P., Tyagi, V., Flusser, J., Ören, T. and Kashyap, R., Eds., Advances in Computing and Data Sciences. ICACDS 2019. Communications in Computer and Information Science, Vol 1046. Springer, Singapore. https://doi.org/10.1007/978-981-13-9942-8 9

[11] Wang, F.H., Pan, J.S. and Jain, L.C. (2009) Discrete Cosine Transform Based Watermarking Scheme and Band Selection. In: Innovations in Digital Watermarking Techniques. Studies in Computational Intelligence, Vol. 232. Springer, Berlin, Heidelberg. https://doi.org/10.1007/978-3-642-03187-8 5

[12] Database (2021). https://www.petitcolas.net/watermarking/image database 